\title{
The nonclassical problem of the compressed bar stability
}

\author{
Khusen Kulterbaev* \\ North Caucasus Federal University, 355017, Stavropol, Russian Federation
}

\begin{abstract}
The non-classical problem of the stability loss of a straight vertical bar with elastic flexible elements at the ends is considered. The mathematical model for the study of stability loss consists of a linearized differential equation for buckling of a constant cross section bar, supplemented by boundary conditions. Critical force is determined by analytical and numerical methods. Two versions of computational algorithms are presented. For one design scheme of the rod in the Matlab computing environment, the critical forces are calculated using two methods. The found critical forces differ insignificantly, which confirms the reliability and efficiency of the proposed algorithms.
\end{abstract}

\section{Introduction}

The phenomenon of stability loss of the equilibrium vertical position since the beginning of Euler's work at the St. Petersburg Academy of Sciences in 1727 has been under constant attention of mathematicians and engineers. Currently, the increased requirements of the scientific and technological revolution and the digital economy have led to a wide variety of used core structures and have complicated this problem. In the last decade, the use of high-strength and lightweight materials in aviation, mechanical engineering and construction has led to the widespread use of thin-walled structures prone to stability loss until the material strength is exhausted. The scientific and technological revolution of our time enhances this tendency, which entails the expansion of research on the problems of sustainability [1 - 6]. At the same time, traditional methods and numerous special cases of determining critical forces using tables and graphs given in technical and reference literature turned out to be ineffective. In unconventional, but already relevant, cases (multispan, variable stiffness, the presence of elastic intermediate supports, etc.), solving the problem of the compressed rods' stability presents significant difficulties, since it is often not possible to establish the spectra of eigenvalues and shapes by analytical methods. These and other reasons have led to the widespread use of numerical methods in calculating building structures [7 - 12].

The complication of structures' design schemes for the above-mentioned reasons led to the need to formulate non-classical problems of the compressed rods' stability. In this work, analytical and numerical methods for determining the critical force are used. A simple universal graphic-analytical method is proposed that allows finding the values of the first

\footnotetext{
*Corresponding author: kulthp@mail.ru
} 
three elements of the spectrum of eigenvalues sufficient for practical needs in the environment of the Matlab computing complex. This method was used in a number of works in the multi-span rods study [13, 14].

\section{Formulation of the problem}

A vertical rod with a hinge support, equipped with two flexible elements in the form of a cylindrical and a spiral spring (Fig. 1), is compressed by a concentrated force F. It is required to determine the critical forces values by the analytical and numerical methods. For this purpose, it is necessary to develop such decision algorithms that will be universal, high-precision and easily implemented in the Matlab computer environment widely used by engineers.

Required:

1. To make a mathematical model of the stability loss of a rectilinear form of equilibrium and the transition to a curvilinear form.

2. To determine the first three values of the critical force, analytically and numerically.

Fig. 1 shows the design scheme in the initial position (solid lines) and after stability loss (dashed lines). The stability loss of the rod will be considered under the following assumptions $[1,2]$ :

1. The axis of the unloaded bar is ideally straight and all external loads and reactions of the supports until stability loss act strictly along this axis.

2. External forces are "dead", i.e., when the bar is deformed, they do not change either in magnitude or in direction.

3. Changes in the geometric dimensions of the bar under subcritical deformations are considered negligible.

4. The relationship between the internal bending moment and the transverse bending of a bar during buckling is described by the usual dependence of the linear theory of bending of beams, based on the hypothesis of flat sections.

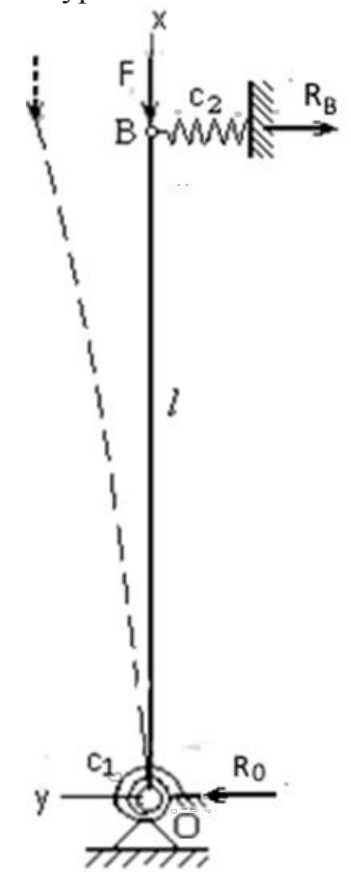

Fig. 1. Design scheme 
It should be emphasized [1], "that the multiplicity of equilibrium positions can be detected only when the equilibrium equations are compiled for a deformed system deviated from its initial unloaded position". This recommendation is taken into account in the following mathematical model.

\section{Mathematical model}

The phenomenon of stability loss consists in the fact that when the longitudinal force $\mathrm{F}$ reaches the critical value $F_{k}$, the rod from the rectilinear vertical equilibrium position goes into an adjacent curved equilibrium position. This phenomenon is called bifurcation. The curvilinear curved axis of the rod after bifurcation is described by the ordinary differential equation of the fourth order

$$
\mathrm{bv}^{\mathrm{IV}}(\mathrm{x})+\mathrm{Fv}^{\prime \prime}(\mathrm{x})=0, \quad \mathrm{~b}=\mathrm{EJ}, \quad \mathrm{x} \in(0, \mathrm{l})
$$

$E$ is the elasticity modulus of the bar material, $J$ is the axial moment of inertia of the cross section, $l$ is the length of the bar, the Roman numeral IV and two dashes in the superscripts correspond to the fourth and second derivatives with respect to the $\mathrm{x}$ coordinate. To obtain a particular equation solution (1), the boundary conditions are added to it at the ends of the rod.

The lower end is hingedly fixed and equipped with a spiral spring. Therefore, there will be three boundary conditions: the deflection is zero, the bending moment is equal to the torsional moment of the spiral spring,

$$
\mathrm{v}(0)=0, \quad \mathrm{bv}^{\prime \prime}(0)=\mathrm{c}_{1} \mathrm{v}^{\prime}(0), \quad \quad \mathrm{bv}^{\prime \prime \prime}(0)=\mathrm{c}_{2} \mathrm{v}(\mathrm{l})
$$

Here $c_{1} v^{\prime}(0)$ is the bending moment created by the spiral spring; $b^{\prime \prime \prime}(0)$ - lateral force; $\mathrm{c}_{2} \mathrm{v}(\mathrm{l})$ is the horizontal support reaction that balances the tensile force of the upper support spring.

The boundary conditions in the upper end section correspond to the bending moment and shear force and have the form.

$$
\begin{gathered}
\mathrm{M}(l)=0 \Rightarrow \mathrm{v}^{\prime \prime}(l)=0, \quad \mathrm{Q}(l)=-\mathrm{Fv}^{\prime}(l)+\mathrm{c}_{2} \mathrm{v}(l) \Rightarrow \mathrm{bv}^{\prime \prime \prime}(l)= \\
=-\mathrm{Fv}^{\prime}(l)+\mathrm{c}_{2} \mathrm{v}(l)=0
\end{gathered}
$$

The equation (1) and additional conditions (2), (3) form a correct mathematical model for determining the critical forces $\mathrm{F}_{\mathrm{k}}$. For this purpose, two fundamentally different solution algorithms will be used.

\section{Solution Algorithm 1}

This refers to the critical forces calculation in a traditional analytical way using computer programs, with a graphical visualization of the counting results on the monitor, according to which the results are set.

We transform the equation (1) to a more convenient form

$$
\mathrm{v}^{\mathrm{IV}}+\mathrm{k}^{2} \mathrm{v}^{\prime \prime}=0, \quad \mathrm{k}^{2}=\frac{\mathrm{F}}{\mathrm{b}}
$$

The general solution to the equation (4) is the function 


$$
v(x)=A_{1} \sin k x+A_{2} \cos k x+A_{3} x+A_{4}
$$

Here $A_{i}$ defines the arbitrary constants of integration to be determined. To determine them, it is necessary to compose a system of linear algebraic equations with a quadratic matrix of the fourth order. Let's write it in matrix-vector form

$$
\mathrm{BA}^{\mathrm{T}}=0
$$

$B$ is a square matrix of the fourth order, the elements of which are determined by the boundary conditions (2), (3), $A$ - column vector $\left(\mathrm{A}_{1}, \quad, \mathrm{~A}_{4}\right)^{\mathrm{T}} T$ is a vector transpose sign. The right side of the system of equations is a null vector.

First, we write out the derivatives of function (5) for an arbitrary point of the rod

$$
\begin{gathered}
\mathrm{v}^{\prime}(\mathrm{x})=\mathrm{A}_{1} \mathrm{kx}-\mathrm{A}_{2} \mathrm{kkx}+\mathrm{A}_{3}, \\
\mathrm{v}^{\prime \prime}(\mathrm{x})=-\mathrm{A}_{1} \sin \mathrm{k}^{2} \sin \mathrm{kx}-\mathrm{A}_{2} \mathrm{k}^{2} \cos \cos \mathrm{kx} \\
\mathrm{v}^{\prime \prime \prime}(\mathrm{x})=-\mathrm{A}_{1} \cos \mathrm{k}^{3} \cos \mathrm{kx}+\mathrm{A}_{2} \mathrm{k}^{3} \sin \sin \mathrm{kx}
\end{gathered}
$$

Let us compose the boundary conditions equations in the sequence as they are given by (2) - (3), then we obtain

$$
\begin{gathered}
\mathrm{A}_{2}+\mathrm{A}_{4}=0,-\mathrm{A}_{1} c_{1} \mathrm{k}-\mathrm{A}_{2} \mathrm{~b} k^{2}-\mathrm{A}_{3} c_{1}=0, A_{1} \sin k l+A_{2} \cos k l=0, \\
\mathrm{~A}_{1} \alpha+A_{2} \beta+\mathrm{A}_{3} \gamma-\mathrm{A}_{4}=0 \\
\alpha=\frac{\mathrm{F}}{\mathrm{c}_{2}} \mathrm{k} \cos \mathrm{k} l-\sin \mathrm{k} l, \quad \beta=-\frac{\mathrm{F}}{\mathrm{c}_{2}} \mathrm{k} \sin \mathrm{k} l-\cos \mathrm{k} l+1, \quad \gamma=\frac{\mathrm{F}}{\mathrm{c}_{2}}-l .
\end{gathered}
$$

Obviously, the system of equations (6) has a trivial solution in the form of a zero vector $\mathrm{A}=0$, which corresponds to the initial rectilinear vertical equilibrium position. This state is of no interest. Of interest are curved deflected positions of the rod, which are unacceptable for the rod to function as a payload-bearing structure. Nonzero solutions of the system of equations (6) can exist only if the determinant of the matrix $B$ is equal to zero, i.e. at

$$
\operatorname{det} B(F)=0
$$

The equation (9) is called characteristic and contains the unknown critical forces $F_{k}, k=$ $1,2,3 \ldots$.

Let us write the equation (6) in the expanded form

$$
\left(\begin{array}{cccc}
0 & 1 & 0 & 1 \\
-c_{1} \mathrm{k} & \mathrm{bk}^{2} & -\mathrm{c}_{1} & 0 \\
\sin \mathrm{k} l & \cos \mathrm{k} l & 0 & 0 \\
\alpha & \beta & \gamma & 0
\end{array}\right)\left(\begin{array}{l}
\mathrm{A}_{1} \\
\mathrm{~A}_{2} \\
\mathrm{~A}_{3} \\
\mathrm{~A}_{4}
\end{array}\right)=\left(\begin{array}{l}
0 \\
0 \\
0 \\
0
\end{array}\right)
$$

The left side of the characteristic equation (9) is a transcendental function and therefore it is very difficult to find its solutions by analytical methods. A simple way out is to plot $F$ $\operatorname{det}(B)$ on a computer and read visually on the monitor the $F$ values at which the curve crosses the horizontal axis. They will be the required critical forces or, in other words, the eigenvalues of the matrix $B$. 
Each critical force has its own form of buckling. In ordinary practice, the solutions of stability problems by their own forms are of little interest or only the first form corresponding to the smallest critical force is determined. But there are special cases where higher critical forces and forms may be of interest. For example, under dynamic shock loads, when in a fast-loading process due to the inertness of the mass, the rod does not have time to deflect when the first critical force is reached. Therefore, the loss of stability occurs already when critical forces of high orders with the corresponding forms are reached. Similar phenomena can occur during seismic and technogenic dynamic impacts on the rod base. These issues require more detailed separate consideration.

Let's look at an example.

Example 1. The rod is made of a steel pipe with a length of $l=7 \mathrm{~m}$ with an outer diameter of $D=108 \mathrm{~mm}$ and a wall thickness of $t=1.4 \mathrm{~mm}$. The modulus of elasticity of the material is $E=2.1 \cdot 10^{11} \mathrm{~N} / \mathrm{m}^{2}$. Cylindrical and spiral springs have stiffness coefficients: $c_{1}=1000 \mathrm{~N} / \mathrm{m}, c_{2}=2000 \mathrm{~N} / \mathrm{rad}$.

According to the solution algorithm given above, a computer program was compiled in the environment of the Matlab computer complex and calculations were performed. The graph of the function F - det, shown in Fig. 2, where bold dots show the intersection of the $\mathrm{F}$ axis. The figure size and its fragments can be increased many times. Therefore, the abscissas of the points are read from a computer monitor with a high degree of accuracy. In this case, the first three critical forces are obtained

$$
\{13,763 ; 29,010 ; 112,698\} \mathrm{kN}
$$

It should be noted that such a computer program makes it easy to study the stability of a rod in its very extensive parameter space.

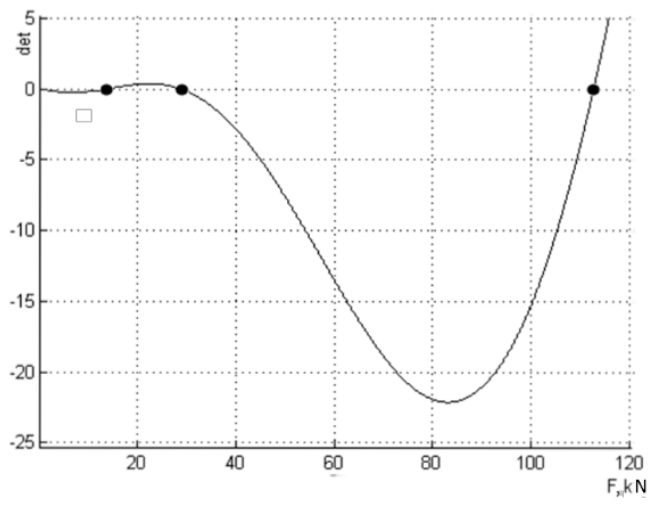

Fig. 2. The calculation results by the analytical method

It is known that in non-classical cases (variable cross-section, multi-span, presence of elastic supports, multi-support, application of several forces, etc.), bibliographic sources are used $[1,2]$ for the particular design schemes. The method proposed here is graphicalanalytical, universal and suitable for determining the critical forces of bars of constant cross-section.

It is not difficult, keeping the problem statement, to adapt the used mathematical model and the solution algorithm to determine the critical forces by the finite difference method [7,12]. For elastic rods with simple geometry, this approach is distinguished by the simplicity and transparency of algorithms and computer programs. A comparative analysis of the two solutions is noteworthy. Therefore, we pass to the corresponding algorithm. 


\section{Solution Algorithm 2}

The main equation (4) in this version remains unchanged. Formulas (2) - (3) will be used, implemented by the methods of the finite difference method. Passing from differential operators to finite-difference operators, we replace the region of continuous variation of the function argument with a uniform grid with $a$ step $h$

$$
\mathrm{L}_{\mathrm{h}}=\left\{\mathrm{x}_{\mathrm{i}}=(\mathrm{i}-1) \mathrm{h}, \mathrm{h}=\mathrm{L} /(\mathrm{n}-1), \mathrm{i}=1,2, \ldots, \mathrm{n}, \mathrm{i}=1,2, \ldots \mathrm{n}\right\} .
$$

A continuous function $\mathrm{v}(\mathrm{x}) \mathrm{s}$ is associated with a grid function $\mathrm{v}_{\mathrm{i}}\left(\mathrm{x}_{\mathrm{i}}\right)$ at the nodes, i. e. $\mathrm{v}_{\mathrm{i}}\left(\mathrm{x}_{\mathrm{i}}\right) \approx \mathrm{v}\left(\mathrm{x}_{\mathrm{i}}\right)$.

The transition from differential operators to finite-difference operators is performed according to formulas that ensure accuracy $\mathrm{O}\left(\mathrm{h}^{2}\right)[9,10]$. The procedure of the finite difference method and elementary transformations give the algebraic system instead of the basic equation (4)

$$
\begin{aligned}
& v_{i-2}+\alpha v_{i-1}+\beta v_{i}+\alpha v_{i+1}+v_{i+2}=0, \\
& i=4,5, \ldots, n-2, \quad \alpha=-4+k^{2} h^{2}, \quad \beta=6-2 k^{2} h^{2}
\end{aligned}
$$

Similar transformations of additional conditions (2), (3) give the following results:

At the lower end

$$
\begin{gathered}
\mathrm{v}_{1}=0, \lambda \mathrm{v}_{1}+\mu \mathrm{v}_{2}+\kappa \mathrm{v}_{3}-\mathrm{v}_{4}=0, \lambda=2+1,5 \mathrm{~s} \mathrm{~h}, \mu=-5+2 \mathrm{~s} \mathrm{~h}, \\
\kappa=4+0,5 \mathrm{~s} \mathrm{~h}, \mathrm{~s}=\mathrm{c}_{1} / 2 \mathrm{~b} . \\
-5 \mathrm{v}_{1}+18 \mathrm{v}_{2}-24 \mathrm{v}_{3}+14 \mathrm{v}_{4}-3 \mathrm{v}_{5}-\xi \mathrm{v}_{\mathrm{n}}=0, \quad \xi=2 \mathrm{~h}^{3} \mathrm{c}_{2} .
\end{gathered}
$$

At the top end

$$
\begin{aligned}
& 3 v_{n-4}-14 v_{n-3}+\rho v_{n-2}+\eta v_{n-1}+\varepsilon v_{n}=0, \\
& \rho=24+\mathrm{Fh}^{2} / \mathrm{b}, \quad \eta=-18-4 \mathrm{Fh}^{2} / \mathrm{b}, \quad \varepsilon=5+3 \mathrm{~h}^{2} \mathrm{~F} / \mathrm{b}-2 \mathrm{c}_{2} \mathrm{~h}^{3} \mathrm{~b} \\
& -v_{n-3}+4 v_{n-2}-5 v_{n-1}+2 v_{n}=0
\end{aligned}
$$

The final result of the transition of the boundary value problem to the grid domain using (12) - (15) is the homogeneous system of algebraic equations

$$
C V=0
$$

where $C$ is a square matrix of order n, $V$ is a column vector representing a continuous function $v(x)$ in the finite difference method. Let us write them out 


$$
\begin{aligned}
& \mathrm{C}=\left(\begin{array}{ccccccccccccccc}
1 & & & & & & \vdots & & & & & & & \\
\lambda & \mu & \kappa & -1 & & & \vdots & & & & & & & \\
-5 & 18 & -24 & 14 & -3 & -\xi & \vdots & & & & & & & \\
& 1 & \alpha & \beta & \alpha & 1 & \vdots & & & & & & & \\
\cdots & \ldots & \ldots & \ldots & \ldots & \ldots & \ldots & \ldots & \ldots & \ldots & \ldots & \ldots & \ldots & \ldots \\
& & & & 1 & \alpha & \beta & \alpha & 1 & & & & & \\
\cdots & \ldots & \ldots & \ldots & \ldots & \ldots & \vdots & \ldots & \ldots & \ldots & \ldots & \ldots & \ldots & \ldots \\
& & & & & & \vdots & & 1 & \alpha & \beta & \alpha & 1 & \\
& & & & & & \vdots & & & 1 & \alpha & \beta & \alpha & 1 \\
& & & & & & \vdots & & & 3 & -14 & \rho & \eta & \varepsilon \\
& & & & & & & & & & & & & \\
& & & & & & \vdots & & & & -1 & 4 & -5 & 2
\end{array}\right), \\
& V=\left(v_{1}, v_{2}, \ldots, v_{n}\right)^{T} .
\end{aligned}
$$

Zero elements are not written out here. The right side of the system of equations (16) is a zero vector.

Obviously, the system of equations (16) is trivial, i.e., zero solution $\mathrm{V} \equiv 0$, which is not of interest in this case. A nontrivial solution can exist only if the determinant of the matrix $C$ is equal to zero, i.e.

$$
\operatorname{det} C(F)=0
$$

The characteristic equation (17) is an algebraic equation of the matrix $C$ and has a set of roots of cardinality $n$. You can do without drawing up this equation, using the possibility of constructing a high-precision graph of the curve of the graph of the det $\mathrm{C}(\mathrm{F})$ function, as in the previous case. The points of intersection of the graph with the $F$ axis will determine the values of the critical forces.

Keeping all the initial data of Example 1, we add to them the parameters of the finitedifference scheme and again determine the eigenvalues, but already the matrix $C$.

Example 2. The rod is made of steel pipe with a length of $l=7 \mathrm{~m}$ with an outer diameter of $D=108 \mathrm{~mm}$ and a wall thickness of $t=1.4 \mathrm{~mm}$. The modulus of elasticity of the material is $E=2.1 \cdot 10^{11} \mathrm{~N} / \mathrm{m}^{2}$. Cylindrical and spiral springs have stiffness coefficients: $c_{1}$ $=1000 \mathrm{~N} / \mathrm{m}, c_{2}=1000 \mathrm{~N} / \mathrm{rad}, n=10001$.

Let's solve the problem in accordance with the above algorithm. The results are shown in Fig. 3 graphically. The first three eigenvalue elements read visually from the monitor screen have meanings:

\section{$\{13.962: \quad 29.215: \quad 112.879\} \mathrm{kN}$}

Obviously, the difference is very small and has no practical significance.

The second conclusion from the solution of the two examples is that the proposed method for determining the critical forces for compressed rods has the property of versatility and is preferable.

According to the two algorithms discussed above, one important conclusion can be drawn, which is that the numerical method can be easily adapted to bars of variable crosssection, while the analytical method for determining the critical force does not have such a possibility. 


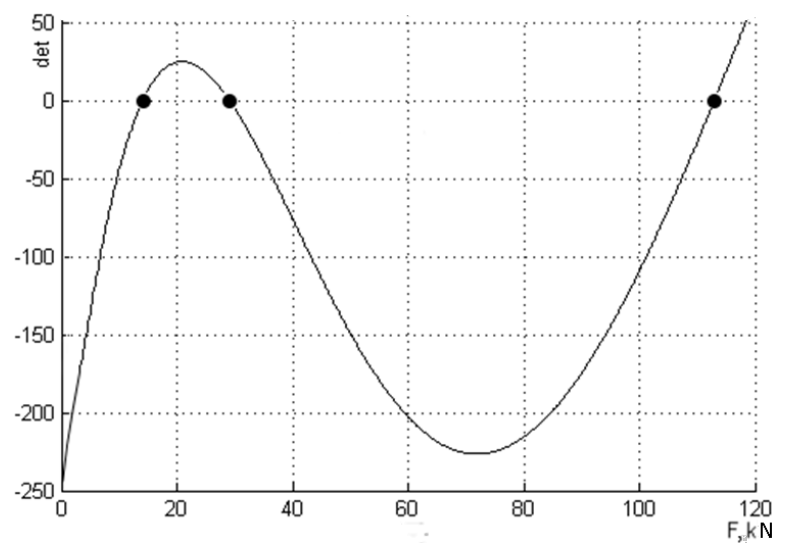

Fig. 3. Results of calculation by the numerical method.

\section{Conclusion}

1. The computing specialized complex Matlab, intended for solving technical problems by mathematical methods, is easily adapted to spectral non-classical problems of structural mechanics.

2. Using the finite difference method, the calculation of critical forces for bars of variable cross-section can be performed using highly accurate universal methods.

\section{References}

1. N.A. Alfutov, The basics of calculating the stability of elastic systems (Mechanical engineering, Moscow, 1978).

2. A.S. Volmir, Stability of deformable systems (Science, Moscow, 1967).

3. A.F. Smirnov, B.Ya. Alexandrov, B.Ya. Laschenikov, N.N. Shaposhnikov, Structural mechanics, Dynamics and stability of structures (Nauka, Stroyizdat, Moscow, 1984).

4. A.M. Maslennikov, Dynamics and stability of structures (Yurayt Publishing House, Moscow, 2016).

5. V.I. Korobko, A.V. Korobko, Dynamics and stability of rod systems (ASV Publishing House, Moscow, 2008).

6. I.A. Birger, Ya.G. Panovko, Strength, stability, vibrations, Handbook in three volumes. Volume 3 (Mechanical engineering, Moscow, 1968).

7. T.D. Karamansky, Numerical methods of structural mechanics (Stroyizdat, Moscow, 1981).

8. V.M. Verzhbitsky, Fundamentals of Numerical Methods (Higher school, Moscow, 2002).

9. P.M. Varvak, L.P. Varvak, The method of meshes in the problems of calculating building structures (Stroyizdat, Moscow, 1977).

10. A.A. Samarskiy, Difference Scheme Theory (Science, Moscow, 1977).

11. V.F. Formalev, D.L. Reviznikov, Numerical methods. 2nd edition (Fizmatlit, Moscow, 2006). 
12. V.P. Ilyin, V.V. Karpov, A.M. Maslennikov, Numerical methods for solving the problems of structural mechanics (Publishing house ASV, Moscow, SPb, SPbSACU, 2005).

13. Kh.P. Kulterbaev, K.A. Karmokov, Bulletin of VolgSACU, Series: Building and architecture 34 (53), 90-98 (2013).

14. T.Yu. Chechenov, On the stability of a multi-span rod on flexible supports, Bulletin of the Nizhniy Novgorod University named after N.I. Lobachevsky, Proceedings of the X All-Russian Congress on fundamental problems of theoretical and applied mechanics 4 (2), 1850-1851 (2011). 